\title{
APROVECHAMIENTO POTENCIAL DE RESIDUOS DE LA AGROINDUSTRIA CALDENSE SEGÚN SU COMPOSICIÓN ESTRUCTURAL
}

\section{POTENTIAL USE OF WASTE FROM CALDENSE AGROINDUSTRY ACCORDING TO ITS STRUCTURAL COMPOSITION}

\author{
Ciliana FLÓREZ MONTES*, Andrés Felipe ROJAS GONZÁLEZ \\ Departamento de Ingeniería Química, Universidad Nacional de Colombia, Sede Manizales, \\ Campus La Nubia, Manizales, Caldas, Colombia.
}

* Autor corresponsal. E-mail: cflorezm@unal.edu.co
Historia del artículo

Recibido: Abril 10, 2018

Evaluado: Agosto 3, 2018

Aceptado: Agosto 13, 2018

Disponible: Septiembre 19, 2018

\section{Resumen}

El presente documento tiene como objetivo la identificación de los principales residuos de la agroindustria en el departamento de Caldas, la caracterización estructural de estos y proponer su aprovechamiento potencial. La caracterización estructural se lleva a cabo mediante la determinación de los componentes estructurales (celulosa, hemicelulosa y lignina) y no estructurales (extractivos y cenizas) de cada uno de los residuos agroindustriales. Al tener en cuenta estos resultados, se identifican las aplicaciones potenciales de los residuos estudiados según sus porcentajes de celulosa, hemicelulosa, lignina y extractivos. Los resultados indican que las semillas de naranja y mandarina, el vástago de tomate de árbol y las cáscaras de mango, guanábana, maracuyá y plátano tienen un aprovechamiento potencial en la industria del papel, la textil, la alimenticia y la de azúcares fermentables, en la fabricación de biomateriales y en la obtención de éter y ésteres de celulosa, debido al porcentaje de celulosa presente en estos. Los residuos con porcentajes importantes de hemicelulosa, como, por ejemplo, las cáscaras de piña y tomate de árbol y las semillas de tomate de árbol, se pueden utilizar en la industria química, alimenticia y farmacéutica. Las cáscaras de mango, guanábana y lulo, así como la borra de café, presentan altos contenidos de lignina, por lo que representan una fuente potencial de compuestos, tales como la vainillina y los lignosulfonatos, los cuales tienen aplicaciones en la industria alimenticia y química. Las cáscaras de maracuyá, piña y mango tienen aplicaciones en la industria alimenticia y farmacéutica debido a su contenido de extractivos.

Palabras clave: celulosa; extractivos; hemicelulosa; lignina; residuos agroindustriales

\section{Abstract}

The aim of this document is to identify the main wastes of the agroindustry in the department of Caldas and, in accordance with its structural characterization, propose a potential use of waste. The structural characterization was carried out by determining the structural components (cellulose, hemicellulose and lignin) and non-structural components (extractives and ash) of each of the agroindustrial wastes. Considering these results, the potential applications of the wastes studied are identified according to their percentages of cellulose, hemicellulose, lignin and extractives. The results indicate that the seeds of orange and tangerine, the stem of tree tomato and the peels of mango, soursop, passion fruit and banana have a potential use in the industry of paper, textile, food and fermentable sugars, in the manufacture of biomaterials and in obtaining cellulose ether and esters, due to the percentage of cellulose present in them. Wastes with significant percentages of hemicellulose, such as pineapple and tree tomato peel and tree tomato seeds, have applications in the chemical, food and pharmaceutical industries. Mango, soursop and lulo peels, and the coffee grounds present high lignin contents, representing a potential source of compounds such as vanillin and lignosulfonates, which have applications in the food and chemical industry. Passion fruit, pineapple and mango peels have applications in the food and pharmaceutical industry due to their extractive content.

Keywords: cellulose; extractives; hemicellulose; lignin; agroindustrial wastes 


\section{INTRODUCCIÓN}

En el departamento de Caldas, los residuos agroindustriales representan un tipo de biomasa lignocelulósica que se genera, principalmente, en procesos tales como la transformación de frutas y verduras, la elaboración de bebidas, la confitería, las conservas y los snacks, entre otros. En la actualidad, los procesos de transformación de la agroindustria caldense generan una gran variedad de residuos, entre los cuales se encuentran las cáscaras de maracuyá, plátano, piña, tomate de árbol, mango, lulo y guanábana, las semillas de tomate de árbol, mandarina y naranja, el vástago de tomate de árbol y la borra de café (Ministerio de Agricultura y Desarrollo Rural, 2006).

Entre las principales agroindustrias del departamento de Caldas se pueden destacar FLP Procesados, Frugy S. A., Passiflora Colombiana S. A., Frutasa, Buencafé liofilizado de Colombia y Comestibles Mapy S. A. S. De estas empresas, es posible obtener residuos como, por ejemplo, cáscara de maracuyá, cáscara de plátano, cáscara de piña, cáscara de tomate de árbol, semilla de tomate de árbol, vástago de tomate de árbol, cáscara de mango, borra de café, cáscara de lulo, semilla de mandarina, semilla de naranja y cáscara de guanábana (Ministerio de Agricultura y Desarrollo Rural, 2006).

En el país se genera una gran cantidad de residuos de frutas (biomasa lignocelulósica), cifra que se puede aproximar a 711.509 toneladas anuales (DANE, 2015). Esta cifra representa el $20.76 \%$ de la producción anual de frutas en el país. En tres industrias de Caldas (Frutasa, Passicol S.A. y Frugy) se generan 2.415 toneladas anuales de residuos (Ministerio de Agricultura y Desarrollo Rural, 2006), lo cual equivale al $27.24 \%$ del fruto fresco consumido por la agroindustria. Si se tiene en cuenta la cantidad de residuos generados solo por estas tres agroindustrias, en el departamento se dispondría de una suma importante de residuos que se pueden aprovechar para diversos fines.

La biomasa lignocelulósica está constituida por componentes estructurales (celulosa, hemicelulosa y lignina) y componentes no estructurales (extractivos y cenizas) (Abril y Navarro, 2012; Cai et al., 2017). La celulosa es el componente principal de las paredes celulares de los vegetales y se define como un homopolímero lineal de cadena larga con alto grado de polimerización y alto peso molecular (Basu, 2010; Barroso, 2010). La hemicelulosa es otro componente de las paredes celulares de las plantas y la conforman cadenas ramificadas de menor grado de polimerización (Basu, 2010). La lignina es un polímero aromático que presenta una estructura tridimensional compleja, se encuentra altamente ramificado y es amorfo (Agyekum y Nyachoti, 2017; Basu, 2010; Barroso, 2010). Los extractivos se definen como compuestos orgánicos de bajo peso molecular presentes en la biomasa que pueden extraerse gracias a la acción de solventes (Ruales, 2015; Almardeai et al., 2017). Se denomina "cenizas" al material resultante de la combustión completa de la biomasa, las cuales están constituidas en mayor medida por $\mathrm{SiO}_{2}$ y $\mathrm{CaO}$, y en menor proporción por óxidos de magnesio, aluminio, potasio y fósforo (Maeda et al., 2017; Melissari, 2012).
El uso principal de la biomasa es su transformación para: 1) la generación de energía, la cual se realiza a través de combustión directa para generar vapor (Galán, 2016; Hupa et al., 2017); 2) procesos termoquímicos para obtener combustibles líquidos, sólidos o gaseosos (Del Val Gento y Giraldo, 2016); 3) procesos biológicos como la digestión anaerobia o la fermentación alcohólica (Do Nascimento et al., 2016); y 4) procesos de transesterificación para la obtención de biodiesel (Arévalo, 2015; Maneerung, 2015). Actualmente, las aplicaciones de la biomasa lignocelulósica se han ampliado debido a las características estructurales propias de cada residuo agroindustrial. Dentro de estas se encuentra la elaboración de diversos productos químicos como, por ejemplo, la vainillina (Harshvardhan et al., 2017), los lignosulfonatos (Chávez-Sifontes y Domine, 2013), el carbón activado (Suhas et al., 2016), los adhesivos (Pradyawong et al., 2017), y los biocombustibles (Demirbas, 2010), entre otros.

La estructura del material lignocelulósico y sus características morfológicas, químicas y estructurales influyen de manera importante en la elección de los procesos en los cuales puede aprovecharse la biomasa agroindustrial. En el caso de las características estructurales, la celulosa se puede utilizar en la industria del papel, en la textil, la alimenticia y la de azúcares fermentables (Carreño et al., 2012), así como en la industria química para la obtención de éteres y ésteres de celulosa (Shokri y Adibkia, 2013). La hemicelulosa se puede emplear como materia prima en diferentes industrias para la generación de diversos productos como el furfural (Campos, 2015), el xilitol (Panesso et al., 2012) y el ácido acético (Sánchez, 2016), entre otros. La lignina presenta diversos usos en la industria, dentro de las cuales se encuentran la obtención de vainillina (Trapero, 2013) y lignosulfonatos. Además, la lignina puede emplearse en la sustitución de resinas de fenol-formaldehído (Suárez, 2010). Debido a la variedad de compuestos extractivos presentes en la biomasa, estos tienen una amplia gama de aplicaciones. Los compuestos bioactivos hacen parte de los extractivos presentes en la biomasa, los cuales, gracias a su capacidad antioxidante, se usan en las industrias farmacéutica, cosmética y alimenticia (Martínez-Valverde et al., 2000).

El objetivo de este trabajo es proponer un aprovechamiento potencial para los residuos generados en la agroindustria caldense. Las alternativas de aprovechamiento se plantean mediante la relación de la información de aprovechamiento presentada en la literatura y la caracterización estructural real de los residuos. Para esto, se seleccionaron y caracterizaron por análisis estructural 12 residuos, generados mediante el procesamiento de algunas frutas en el departamento de Caldas.

\section{MATERIALES Y MÉTODOS}

Los residuos provenientes de la agroindustria caldense seleccionados para este estudio se caracterizan mediante análisis de composición química (celulosa, hemicelulosa, lignina y extractivos), materia seca y cenizas, al seguir la metodología que se describe a continuación. 


\section{Selección y obtención de los residuos agroindustriales}

Los residuos agroindustriales se seleccionan según su disponibilidad en el departamento de Caldas, específicamente en la subregión centro sur. Se seleccionaron seis empresas: FLP Procesados, Frugy S.A., Passiflora Colombiana S.A. y Frutasa, las cuales procesan una gran variedad de frutas, además de Buencafé Liofilizado de Colombia y Comestibles Mapy S.A.S. que procesan café y plátano, respectivamente. Con base en la variedad de empresas y residuos generados en ellas, se identificaron 12 residuos principales: cáscara de maracuyá (CMr), cáscara de plátano (CP), cáscara de piña (CPñ), cáscara de tomate de árbol (СTa), semilla de tomate de árbol (sTa), vástago de tomate de árbol (VTa), cáscara de mango (CMg), borra de café $(\mathrm{BC})$, cáscara de lulo $(\mathrm{CL})$, semilla de mandarina (SMd), semilla de naranja (SN) y cáscara de guanábana (CGu).

\section{Pretratamiento de los residuos agroindustriales}

La preparación de los residuos para el análisis de composición estructural se realiza de acuerdo con la norma NREL/TP-51042620. Los residuos se almacenan a $-20^{\circ} \mathrm{C}$ hasta el momento de realizar el análisis estructural. Para este análisis los residuos se reducen de tamaño al utilizar una cortadora de cuchillas, y se someten a un presecado a $45^{\circ} \mathrm{C}$ hasta peso constante. Finalmente, los residuos presecados se vuelven a moler hasta tamaños menores a $1 \mathrm{~mm}$ mediante un molino de discos.

\section{Caracterización estructural de los residuos agroindustriales}

La composición estructural de los residuos se determina mediante la cuantificación de los compuestos extractivos (EXT), lignina (LGN), celulosa (CEL), hemicelulosa (HMC) y compuestos inorgánicos (cenizas). El contenido de materia seca (MS) se determina mediante la norma ASTM E1756-01, y para la determinación de cenizas (CZ) se aplica la norma ASTM E1755-01. Para cuantificar los porcentajes de CEL, HMC y LGN los residuos se someten previamente a extracciones Soxhlet, como indica la norma NREL/TP510-42619. El porcentaje de lignina ácido insoluble (LGN) o lignina de Klason se establece según la norma NREL/TP-510-42618. En la determinación del porcentaje de holocelulosa (HLC) se sigue la norma ASTM D1104 y la cuantificación del porcentaje de celulosa se realiza según la metodología de Han y Rowell (1997). Estos análisis se realizan por triplicado.

\section{Análisis del aprovechamiento potencial de los residuos}

El aprovechamiento potencial de los residuos se plantea teniendo en cuenta los resultados obtenidos de la caracterización estructural (porcentajes de celulosa, hemicelulosa, lignina y extractivos) de los residuos agroindustriales. La caracterización estructural aporta la información básica para establecer el aprovechamiento potencial de los residuos de la agroindustria caldense en industrias como la química, textil, papelera, farmacéutica o alimenticia.

\section{RESULTADOS Y DISCUSIÓN |}

Las empresas FLP Procesados, Frugy S.A., Passiflora Colombiana S.A., Frutasa, Buencafé Liofilizado de Colombia y Comes- tibles Mapy S.A.S. se seleccionan teniendo en cuenta que estas se centran en la transformación de materias primas provenientes del sector agrícola, como, por ejemplo, maracuyá, plátano, piña, tomate de árbol, mango, café, lulo, mandarina, naranja y guanábana. Estas materias primas son representativas en el departamento de Caldas (Cardona et al., 2012) y pueden generar una cantidad importante de residuos agroindustriales, entre los que se encuentran cáscaras, semillas, vástagos y borra. Los residuos mencionados pueden tener aplicaciones para la obtención de productos de interés en la industria, por tanto, conocer las características estructurales propias de cada uno de estos permite determinar el aprovechamiento adecuado, tal como se presenta a continuación.

\section{Caracterización estructural de los residuos agroindustriales}

En la (Tabla 1) se presentan los resultados obtenidos para materia seca, cenizas, extractivos en agua y en etanol y holocelulosa. Según estos resultados, se observa que los porcentajes de MS oscilan entre $11 \%$ y $42 \%$, siendo la CMr el residuo con menor contenido de MS y la $\mathrm{SN}$ el residuo con mayor contenido de MS. En cuanto al contenido de cz, se puede observar que la BC presenta el menor porcentaje $(0,46 \%)$ y el vTa el mayor porcentaje (12,80\%).

Tabla 1. Caracterización estructural de los residuos agroindustriales.

\begin{tabular}{|c|c|c|c|c|c|}
\hline RESIDUO & $\% \mathbf{M S}$ & $\% \mathrm{CZ}$ & $\% \mathrm{EXT}_{\mathrm{AGUA}}$ & $\%$ EXT $_{\text {ETANOL }}$ & $\% \mathrm{HLC}$ \\
\hline $\mathrm{CMr}$ & 11.34 & 8.03 & $62.69 \pm 0.65$ & $4.84 \pm 0.24$ & $58.35 \pm 0.19$ \\
\hline $\mathrm{cp}$ & 13.78 & 8.94 & $38.60 \pm 0.06$ & $7.01 \pm 0.27$ & $71.98 \pm 0.17$ \\
\hline CPñ & 14.52 & 4.81 & $57.99 \pm 1.22$ & $11.81 \pm 0.14$ & $72.92 \pm 0.16$ \\
\hline ста & 17.04 & 8.67 & $50.28 \pm 1.52$ & $10.05 \pm 0.6$ & $84.97 \pm 0.37$ \\
\hline STa & 21.09 & 4.03 & $35.04 \pm 0.26$ & $22.37 \pm 0.24$ & $57.80 \pm 0.03$ \\
\hline vта & 18.33 & 12.80 & $26.75 \pm 0.23$ & $5.51 \pm 0.01$ & $57.62 \pm 0.24$ \\
\hline CMg & 21.87 & 2.80 & $59.82 \pm 0.45$ & $8.06 \pm 0.33$ & $59.57 \pm 0.11$ \\
\hline bc & 30.89 & 0.46 & $14.56 \pm 0.15$ & $14.37 \pm 0.17$ & $60.11 \pm 0.18$ \\
\hline $\mathrm{cl}$ & 14.79 & 6.06 & $53.95 \pm 0.52$ & $3.85 \pm 0.10$ & $58.45 \pm 0.16$ \\
\hline SMd & 33.26 & 1.92 & $30.65 \pm 0.71$ & $27.78 \pm 0.54$ & $65.99 \pm 0.27$ \\
\hline sn & 42.60 & 3.26 & $21.49 \pm 0.62$ & $32.02 \pm 0.80$ & $65.41 \pm 0.32$ \\
\hline $\mathrm{CGu}$ & 31.98 & 6.22 & $42.88 \pm 0.50$ & $4.09 \pm 0.03$ & $57.20 \pm 0.37$ \\
\hline
\end{tabular}




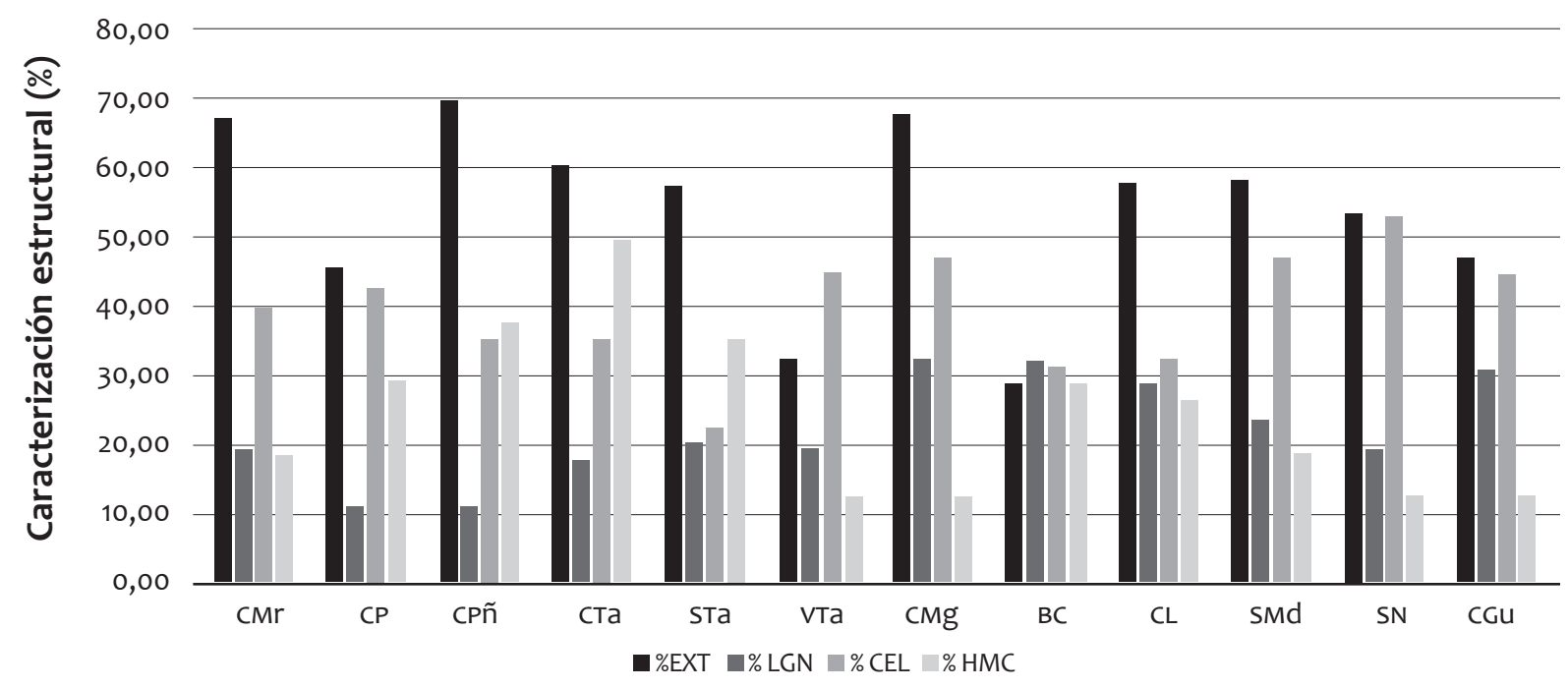

Figura 1. Caracterización estructural de los residuos agroindustriales en base libre de extractivos.

En la (Fig. 1) se presenta un diagrama de barras con la caracterización estructural en base libre de extractivos de cada uno de los residuos. Al observar el porcentaje de EXT de cada uno de los residuos agroindustriales, se tiene que estos valores varían entre el $28 \%$ y el $69 \%$, por lo que podría esperarse que estos residuos presenten una cantidad considerable de proteínas, aceites, polisacáridos y sales, entre otros componentes que puedan separarse al emplear disolventes (Basu, 2010; Sandak et al., 2017).

Es esta figura también se puede observar que el porcentaje de LGN varía entre el $10 \%$ y el $32 \%$, siendo la CMg y la BC los residuos con mayor contenido de LGN. El porcentaje de HLC se encuentra entre el $57 \%$ y el $65 \%$ para la mayoría de los residuos, aunque tres de estos superan dicho porcentaje al alcanzar valores de LGN del $71,98 \%$, el $72,92 \%$ y el $84,97 \%$, para la CP, CPñ y CTa, respectivamente. Para la CEL esta varía entre el $30 \%$ y el $45 \%$ para casi todos los residuos. La STa a tiene un porcentaje de CEL menor al 30\%, mientras que para la SMd $(47,03)$, la CMg $(47,37)$ y la SN $(53,26)$ este porcentaje es mayor al $47 \%$. Finalmente, los porcentajes de HMC varían desde el $12 \%$ hasta valores del $49 \%$. En consecuencia, se puede decir que, en general, todos los residuos analizados presentan una caracterización estructural muy variada, por lo que se espera que estos tengan diversas aplicaciones.

En cuanto a componentes estructurales, la mayoría de los residuos analizados están constituidos en mayor medida por CEL, lo que indica que tienen una estructura cristalina en su pared celular $\mathrm{y}$, por tanto, se espera que muestren una alta resistencia mecánica (Kumar et al., 2017). Los residuos de CPñ, Ста у STa presentan un contenido significativo de HMC, lo que genera una menor resistencia mecánica, ya que su pared celular posee una estructura amorfa (Cruz et al., 2018). La LGN es el componente estructural con los porcentajes más bajos, tal como se espera, debido a que este componente representa, por lo general, entre el $18 \%$ y el $25 \%$ de la biomasa (Basu, 2010).
En la (Fig. 2) se muestra la relación de hemicelulosa a lignina con respecto a la relación de celulosa a lignina. Si se tienen en cuenta los valores presentados en esta figura se puede tener un estimado del rendimiento de la pirólisis para estos residuos. En esta figura también se observa cómo los residuos que se encuentran sobre la línea delgada son aquellos que presentan un mayor porcentaje de HMC, por esto se esperaría que durante la pirólisis de STa, CTa, CPñ y CP se produzcan gases no condensables y poca cantidad de alquitrán (Basu, 2010).

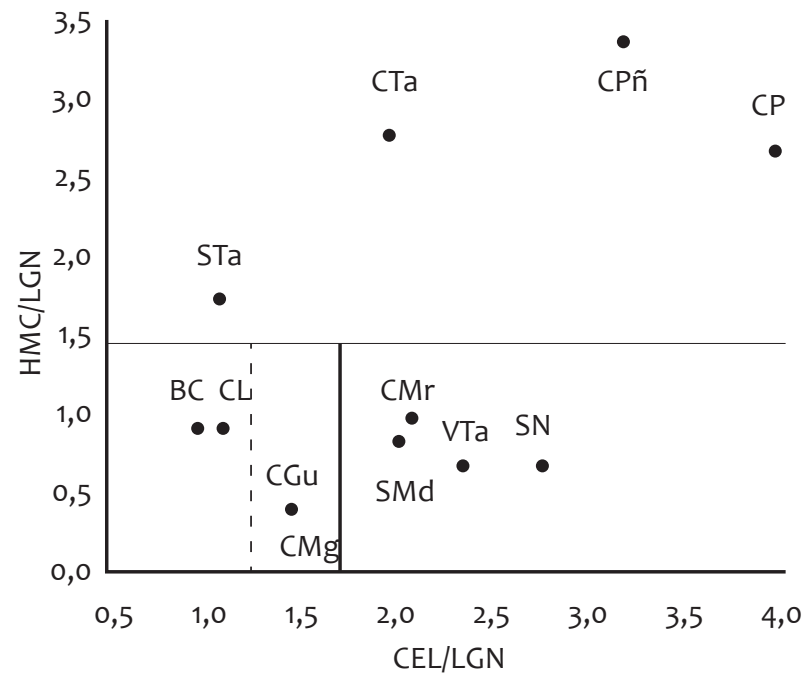

Figura 2. Relación de constituyentes estructurales de los residuos.

Los residuos con mayor porcentaje de CEL se ubican en la parte inferior a la derecha de la línea punteada (Fig. 2). Estos residuos son VTa, CMg, SMd, SN, CGu y Mr, los cuales, a su vez, 
presentan el menor porcentaje de HMC. Para estos residuos se espera que durante la pirólisis se generen gases condensables y una cantidad insignificante de carbonizado (Basu, 2010). En la parte inferior, a la izquierda de la línea gruesa (Fig. 2), se encuentran los residuos con mayor porcentaje de LGN, los cuales son CMg, BC, CL y CGu. La pirólisis de estos residuos producirá entonces una cantidad considerable de compuestos aromáti$\cos$ y carbonizados, mientras que su producto gaseoso es mínimo. De igual forma, la pirólisis de la lignina contribuye al rendimiento del líquido, el cuál contendrá bioaceites y alquitrán (Basu, 2010).

\section{Aprovechamiento potencial de los residuos agroindustriales}

Los residuos con mayor porcentaje de EXT son las CMr, las CPñ y las CMg, aunque, en general, todos los residuos presentan una cantidad considerable de este componente no estructural, pues la gran mayoría de estos compuestos se requieren en cantidades muy pequeñas para cumplir funciones fisiológicas de gran importancia (Prinsen, 2010; Aragonès et al., 2017). Los compuestos bioactivos son un ejemplo de extractivos que, incluso en cantidades mínimas, presentan beneficios en la salud, y son, principalmente, antioxidantes (Martínez-Valverde et al., 2000). Algunos de los compuestos que pueden estar presentes en los residuos son los ácidos fenólicos, flavonoides, lignanos y taninos, los cuales capturan los radicales libres presentes en el cuerpo (Barbosa, 2013), relacionados con el deterioro de las células y el envejecimiento (Coronado et al., 2015; Jiménez-Mora y Rodríguez-Romero, 2018).

Al tener en cuenta los resultados obtenidos en la caracterización estructural, se construye la (Tabla 2), en la cual se presenta una matriz con las posibles aplicaciones de cada uno de los residuos agroindustriales.

Según la (Tabla 2), los residuos como SN, vTa, CMg, SMd, CGu, CMr y CP que tienen un alto contenido de CEL, podrían utilizarse como materia prima en una gran variedad de procesos, entre los que se destacan la industria del papel, la textil, la alimenticia y los azúcares fermentables, así como en la fabricación de biomateriales (Álvarez et al., 2012; Carreño et al., 2012). Estos residuos también podrían derivarse en éteres y ésteres, los cuales tienen usos como espesantes, estabilizantes, formadores de película, emulsificantes, agentes de suspensión, ligantes y como base para películas fotográficas, filtros de cigarrillos, marcos de gafas, juguetes y mangos de herramientas (Pérez et al., 2013; Shokri y Adibkia, 2013). El acetato de celulosa se usa en lacas, películas fotográficas, cinematográficas, RX y fibras (Pérez et al., 2013). La metilcelulosa se emplea como espesante en la industria farmacéutica, en la alimenticia, la textil y la de detergentes; la carboximetilcelulosa se utiliza como espesante y estabilizador en la suspensión de detergentes; y la dietilaminoetilcelulosa tiene aplicaciones como estabilizante en dispersiones (Sanz, 2016).

De acuerdo con la (Tabla 2), las CPñ, las CTa y las STa, residuos agroindustriales con un alto contenido de HMC, podrían ser aprovechados para la producción de furfural. El furfural es una plataforma de gran importancia en la industria química, pues a partir de este se elaboran productos tales como alcohol furfurílico, furfurilamina, ácido furoico y ácido furanacrílico, furano y tetrahidrofurano (Campos, 2015; Chatterjee et al., 2018). De igual forma, estos residuos tienen potencial como materia prima en las industrias alimentaria, farmacéutica y química, ya que con la HMc es posible producir ácido acético, compuesto ampliamente empleado en dichas industrias (Sánchez, 2016).

Si se tiene en cuenta que tanto la CEL como la HMC pueden ser hidrolizadas y sometidas a procesos fermentativos, los residuos con porcentajes altos de estos componentes estructurales tienen potencial en la industria de alcoholes. Una de las aplicaciones principales es la producción de bioetanol, que es uno de los biocombustibles de segunda generación más estudiados en los últimos años (Albarracín, 2015).

Al tomar como referencia la (Tabla 2), las CMg, BC, CGu y CL, que presentan una cantidad importante de lignina, pueden utilizarse para sintetizar vainillina, compuesto que es ampliamente usado en la industria alimentaria y farmacéutica por sus propiedades organolépticas (Trapero, 2013). Según el contenido de LGN, estos residuos agroindustriales también tienen un potencial aprovechamiento en la obtención de lignosulfonatos, los cuales tienen gran importancia en la industria de fertilizantes, plastificantes, dispersantes y emulsionantes (Pedraja, 2016). Los residuos con altos contenido de LGN no son recomendables para procesos fermentativos, ya que esta estructura constituye una barrera física al momento del pretratamiento de la hidrólisis ácida y enzimática, pues dificulta la penetración del ácido o las enzimas celulósicas (Álvarez et al., 2012).

Tabla 2. Aplicaciones potenciales de los residuos agroindustriales.

\begin{tabular}{|c|c|c|c|c|c|c|c|}
\hline RESIDUO & $\begin{array}{l}\text { XIL- } \\
\text { ITOL }\end{array}$ & PAPEL & $\begin{array}{l}\text { ÁCIDO } \\
\text { ACÉTICO }\end{array}$ & ETANOL & $\begin{array}{l}\text { ACETATO } \\
\text { DE CELU- } \\
\text { LOSA }\end{array}$ & $\begin{array}{l}\text { FURFU- } \\
\text { RAL }\end{array}$ & $\begin{array}{c}\text { VAINIL } \\
\text { LIA }\end{array}$ \\
\hline CMr & & $\mathrm{x}$ & & $x$ & $x$ & & \\
\hline$C P$ & & $\mathrm{x}$ & & $\mathrm{x}$ & $\mathrm{x}$ & & \\
\hline CPñ & $x$ & & $x$ & & & $\mathrm{x}$ & \\
\hline ста & & $\mathrm{x}$ & $\mathrm{x}$ & & & $\mathrm{x}$ & \\
\hline sтa & & & $\mathrm{x}$ & & & $x$ & \\
\hline vта & & $x$ & & $x$ & $x$ & & \\
\hline CMg & $x$ & & & $x$ & $\mathrm{x}$ & & $x$ \\
\hline$B C$ & $\mathrm{x}$ & & & & & & $\mathrm{x}$ \\
\hline $\mathrm{CL}$ & $x$ & & & & & & $x$ \\
\hline SMd & & $x$ & & $x$ & $\mathrm{x}$ & & \\
\hline SN & & $\mathrm{x}$ & & $\mathrm{x}$ & $\mathrm{x}$ & & \\
\hline cGu & $\mathrm{x}$ & $\mathrm{x}$ & & & $\mathrm{x}$ & & $\mathrm{x}$ \\
\hline
\end{tabular}

El eugenol es un compuesto fenólico generado mediante la degradación térmica de la lignina (este se conoce como esencia de clavo). El eugenol presenta diversos usos por sus propiedades farmacológicas, este compuesto se emplea en estomatología y en preparaciones higiénicas orales. También 
tiene aplicaciones como saborizante, anestésico tópico, protector dental y desinfectante en la obturación de conductos radiculares, entre otros (González, 2002).

En la actualidad, la cMr tiene gran potencial para la obtención de pectina, por lo cual sería necesario evaluar el porcentaje de este componente estructural de la pared celular presente en dicho residuo. La pectina es un producto de gran interés en la industria alimenticia debido a que aporta textura y consistencia, por lo que se utiliza en la elaboración de mermeladas, jaleas, concentración de bebidas, zumo de frutas, productos lácteos fermentados y directamente acidificados, productos lácteos gelificados y golosinas (Rentería, 2014). Si se tiene en cuenta el alto contenido de EXT de la CMr, este residuo podría usarse en la obtención de proteínas y derivados hidrosolubles, que tienen propiedades antioxidantes, antihipertensivas e inmunomoduladores (Sánchez-Mendoza et al., 2016).

Para la CP también se ha estudiado la obtención de pectinas de bajo metoxilo (Cabarcas et al., 2012). Este tipo de pectina puede formar geles en ausencia de azúcares y en presencia de calcio, por lo que tiene aplicaciones en la elaboración de alimentos bajos en calorías y dietéticos (Chasquibol et al., 2008).

El contenido de HEM presente en la CPñ permite el uso de este residuo en la obtención de xilitol, el cual tiene un poder edulcorante similar a la sacarosa, por tanto, es un producto dirigido, principalmente, a las personas diabéticas. El xilitol se obtiene mediante la hidrogenación catalítica de la D-xilosa, la cual se puede obtener bajo condiciones sencillas de hidrólisis del residuo (Ramírez et al., 2012).

De acuerdo con el contenido de EXT etanol las STa presentan un contenido graso importante, por lo cual este residuo se convierte en una materia prima que puede utilizarse para la elaboración de productos destinados a la alimentación humana (BelénCamacho et al., 2004). Además, al considerar el contenido de HMC de las STa, este residuo podría utilizarse en la obtención de ácido acético, compuesto ampliamente empleado en la industria alimenticia, farmacéutica y química (Sánchez, 2016).

Los residuos con alto contenido de CEL como el vTa, la CTa y la CMg tienen potencial en la industria de elaboración de papel, debido a que su estructura básica es un entramado de fibras de celulosa que presentan una alta resistencia a la tracción y absorben los aditivos manejados en la elaboración del papel. Además, son flexibles, estables y de color blanco. Por otra parte, la CMg presenta porcentajes de LGN superiores al 30\%, por ende, no se recomienda en la elaboración de papel, ya que sería necesario el manejo de procesos químicos para retirar este componente (Gonzáles et al., 2016). Si se considera el contenido de CEL de la CMg, este se convierte en una materia prima viable para la extracción de metabolitos fermentables para la obtención de alcoholes, como, por ejemplo, el etanol, mediante hidrólisis térmica seguida de una hidrólisis enzimática (Mejía et al., 2007).

La BC y la CL presentan un alto contenido de LGN, por lo que tienen potencial interés en la obtención de lignosulfonatos, los cuales se usan como agentes ligantes en la industria de fertilizantes en forma de pellets. También tienen aplicaciones como plastificantes de hormigón y en la producción de ladrillos y materiales cerámicos. Por otra parte, los lignosulfonatos se emplean como dispersantes y emulsionantes, auxiliares de molienda, fluidos de perforación de pozos petrolíferos, agentes de curtido, fabricación de tablas de yeso y fabricación de resinas fenólicas (Pedraja, 2016).

Debido al porcentaje de CEL presente en las CGu, las SMd y las SN, estos residuos se pueden aprovechar para la obtención de acetato de celulosa (Prinsen, 2010). Asimismo, podrían utilizarse en la elaboración de biomateriales como termoestables y cemento, haciendo uso de fibras de celulosa (Álvarez et al., 2012). Por otra parte, las semillas de cítricos (como las SMd y las SN) presentan un alto contenido de $\mathrm{EXT}_{\text {etanol }}$, por lo que representan una fuente de materiales oleaginosos con características deseables para el consumo humano. Estos aceites contienen, principalmente, triglicéridos, de modo que a partir de estos residuos se podría obtener una variedad de ácidos grasos esenciales como el linoleico, oleico, linolénico, palmítico y esteárico (Londoño-Londoño et al., 2012).

Tal como se presentó, los residuos agroindustriales son materiales aptos para su aprovechamiento mediante la obtención de productos de interés con valor económico o social (Saval, 2012). Sin embargo, la inadecuada disposición de los residuos agroindustriales es uno de los principales problemas que presenta la agroindustria, debido a que estos tienen un impacto negativo en el medio ambiente, como consecuencia de su alta concentración de materia orgánica (Peñaranda et al., 2017). Por tanto, la producción de este tipo de residuos genera no solo alteraciones en el medio ambiente, sino que también puede conllevar a pérdidas económicas asociadas a los altos costos de almacenamiento, tratamiento, transporte y disposición de los residuos agroindustriales (Vargas y Pérez, 2018).

Si se considera lo anterior, el aprovechamiento de los residuos agroindustriales posibilita la solución de problemáticas ambientales y económicas y, de esta forma, se disminuye el uso de recursos naturales como materia prima para la generación de ciertos productos (Vargas y Pérez, 2018). A fin de seleccionar una alternativa adecuada de aprovechamiento de los residuos agroindustriales es importante conservar sus propiedades, ya que estos se exponen a plagas, insectos, roedores y factores climáticos que causan su contaminación (Saval, 2012; Yepes et al., 2008). De igual forma, están expuestos a agentes microbiológicos que causan reacciones de descomposición o la generación de compuestos tóxicos (Muñoz-Muñoz et al., 2014). En consecuencia, los residuos agroindustriales se pueden someter a un proceso de esterilización y secado o deshidratación con el fin evitar reacciones de descomposición (Alzate et al., 2011), y deben almacenarse de tal forma que se evite el contacto con plagas, insectos y roedores, y a bajas temperaturas.

\section{CONCLUSIONES}

Las agroindustrias FLP procesados, Frugy S.A., Passiflora Colombiana S.A., Frutasa, Buencafé liofilizado de Colombia y 
Comestibles Mapy S.A.S., son una fuente potencial de residuos, de los cuales, las cáscaras de mango y maracuyá son los residuos que se generan en mayor proporción. Estos residuos pueden utilizarse en diferentes procesos de la industria, lo cual influirá de forma positiva en aspectos ambientales, económicos y sociales en el departamento.

Según la caracterización estructural de los residuos estudiados, se encontró que, en cuanto a componentes estructurales, la mayoría de los residuos están compuestos en mayor medida por CEL, seguido por HMC y LGN. En general, todos los residuos agroindustriales presentan contenidos considerables de EXT, los cuales incluso en bajas cantidades presentan un alto valor agregado por los importantes beneficios que aportan a la salud humana; por esto, los residuos pueden utilizarse para la extracción de compuestos extractivos específicos.

Se espera que los residuos estudiados tengan un comportamiento similar a la biomasa proveniente de la madera por su contenido de CEL. Por ende, la pirólisis de estos generará gases condensables y mínimas cantidades de carbón.

Los altos contenidos de CEL en residuos como SN, VTa, CMg, SMd, CGu, CMr y CP hacen que estos tengan un potencial aprovechamiento en la industria del papel, en la textil, la alimenticia y la de azúcares fermentables, en la fabricación de biomateriales y en la obtención de éter y ésteres de celulosa. Los residuos ricos en HMC, como lo son las CPñ, las ста y las Sтa tienen amplias aplicaciones en la industria química, alimenticia y farmacéutica.

La LGN presente en CMg, BC, CGu y CL los convierte en una fuente potencial de compuestos como la vainillina, utilizada en la industria alimentaria y farmacéutica, o los lignosulfonatos, que tienen usos en la industria de fertilizantes, plastificantes, dispersantes y emulsionantes. La aplicación de residuos con un alto contenido de LGN en procesos fermentativos no es recomendable, dado que esta estructura representa una barrera física que dificulta el acceso del ácido o las enzimas celulósicas en el momento de la hidrólisis.

Teniendo en cuenta el alto porcentaje de EXT que presentan los residuos, estos pueden considerarse como fuente potencial de compuestos bioactivos, tales como proteínas, aceites esenciales y polisacáridos, entre otros, los cuales tienen propiedades benéficas para la salud, por lo que pueden utilizarse en la industria alimenticia, en la farmacéutica y la cosmética.

\section{AGRADECIMIENTOS |}

Los autores agradecen a la Universidad Nacional de Colombia, sede Manizales, por el apoyo económico al proyecto titulado "Identificación, cuantificación y caracterización de los residuos orgánicos generados en la agroindustria caldense para su aprovechamiento energético y bioactivos", con código Hermes 35961 , y a las empresas que nos proporcionaron los residuos.

\section{REFERENCIAS}

- Abril, A. y Navarro, E. (2012). Etanol a partir de biomasa lignocelulósica (1ªed.). Andalucía: Aleta Ediciones.

- Agyekum, A. K. y Nyachoti, C. M. (2017). Nutritional and Metabolic Consequences of Feeding High-Fiber Diets to Swine: A review. Engineerin, 3(5),716725. doi https://doi.org/10.1016/J.ENG.2017.03.010.

- Albarracín, K. G., Jarmillo, L. I. y Albuja, M. (2015). Obtención de bioetanol anhidro a partir de paja (Stipa ichu). Revista Politécnica, 36(2), 109-117. doi: https://doi.org/10.1016/J.ENG.2017.03.010.

- Almardeai, S., Bastidas-Oyanedel, J. R., Haris, S. y Schmidt, J. E. (2017). Avicennia marina Biomass Characterization towards Bioproducts. Emirates Journal of Food and Agriculture, 29(9), 710-715. doi: https://doi.org/10.9755/ ejfa.2017.v29.ig.109.

- Álvarez, A., Salgado, R., García, E., Domínguez, M. M., Granados, J. M., Aguirre, A. ... y Mendoza, A. M. (2012). Aprovechamiento integral de los materiales lignocelulósicos. Revista Iberoamericana de Polímeros, 13(4), 140150. Recuperado de http://www.ehu.eus/reviberpol/pdf/SEPT12/alvarez.pdf.

- Alzate, L. M., Jiménez, C. y Londoño, J. (2011). Aprovechamiento de residuos agroindustriales para mejorar la calidad sensorial y nutricional de productos avícolas. Producción + limpia, 6(1), 108-127. doi: http://www.scielo.org.co/pdf/ $\mathrm{pml} / \mathrm{v} 6 \mathrm{n} 1 / \mathrm{v} 6 \mathrm{n} 1 \mathrm{a} 10 . \mathrm{pdf}$.

- Aragonès, G., Danesi, F., Del Rio, D. y Mena. P. (2017). The Importance of Studying Cell Metabolism when Testing the Bioactivity of Phenolic Compounds. Trends in Food Science \& Technology, 69, 230-242. doi: https://doi. org/10.1016/j.tifs.2017.02.001.

- Arévalo, W. (2015). La biomasa: una alternativa energética proveniente de la vida misma. En L. A. Montenegro-Mora (Ed.), Las energías sustentables y sostenibles en el departamento de Nariño (pp. 80-90). Nariño: Unimar.

- Barbosa, L. (2013). Desarrollo y evaluación de nuevos sistemas de envases activos de uso alimentario (Tesis de doctorado en Nutrición y Bromatología). Universidad de Santiago de Compostela, Santiago de Compostela.

- Barroso, M. (2010). Pretratamiento de biomasa celulósica para la obtención de etanol en el marco de una biorrefinería (Tesis de pregrado en Ingeniería Forestal). Universidad Politécnica de Madrid, Madrid. Recuperado de http:// oa.upm.es/10559/1/MIGUEL_BARROSO_CASILLAS.pdf.

- Basu, P. (2010). Biomass Characteristics. En P. Basu (Ed.), Biomass Gasification and Pyrolysis. Practical Design and Theory (pp. 27-63). Kidlington: Elsevier. doi: https://doi.org/10.1017/CBO9781107415324.004.

- Belén-Camacho D. R., Sánchez, E. D., García, D., Moreno-Álvarez, M. J. y Linares, O. (2004). Características fisicoquímicas y composición en ácidos grasos del aceite extraído de semillas de tomate de árbol (Cyphomandra betacea Sendt) variedades roya y amarilla. Grasas y Aceites, 55(4), 428-433. Recuperado de http://www.ingentaconnect.com/content/doaj/00173495/200 4/00000055/00000004/art00013.

- Cabarcas, E., Guerra, A. F. y Henao, C. A. (2012). Extracción y caracterización de pectina a partir de cáscaras de plátano para desarrollar un diseño general del proceso de producción (Tesis de pregrado). Programa de Ingeniería Química, Universidad de Cartagena, Cartagena de Indias. doi: http://190.242.62.234:8080/jspui/bitstream/11227/109/1/Trabajo\%20de\%20 grado-Extraccion $\% 20 y \% 20$ caracterizacion $\% 20$ de $\% 20$ pectina $\% 20$ apartir $\% 20$ de $\% 20$ cascaras $\% 20$ de $\% 2$ platano $\% 20$ para $\% 2$ desarrollar $\% 20$ un $\% 20$ dise $\% C_{3}$ \%B10\%2ogenera 1.pdf.

- Cai, J., He, Y., Yu, X., Banks, S. W., Yang, Y., Zhang, X. ... y Bridwater, A. (2017). Review of Physicochemical Properties and Analytical Characterization of Lignocellulosic Biomass. Renewable and Sustainable Energy Reviews, 76, 309322. doi: https://doi.org/10.1016/j.rser.2017.03.072.

- Campos, M. J. (2015). Nuevos procesos catalíticos para la obtención de furfural (Tesis de doctorado). Doctorado en Química Física Aplicada, Universidad Autónoma de Madrid, Madrid. doi: https://doi.org/10.1016/j.rser.2017.03.072.

- Cardona, C. A., Orrego, C. E. y Tamayo, J. A. (2012). Arcano, una oportunidad para el desarrollo de la agroindustria en el departamento de Caldas (1ra ed.). Manizales: Universidad Nacional de Colombia. 
- Carreño, L. C., Caicedo, L. A. y Martínez, C. A. (2012). Técnicas de fermentación y aplicaciones de la celulosa bacteriana: una revisión. Ingeniería y Ciencia, 8(16):307-335. doi: http://www.scielo.org.co/pdf/ince/v8n16/v8n16a12.pdf.

- Chasquibol, N., Arroyo, E. y Morales, J. C. (2008). Extracción y caracterización de pectinas obtenidas a partir de frutos de la biodiversidad peruana. Ingeniería Industrial, 26, 175-199. doi: http://repositorio.ulima.edu.pe/handle/ulima/2438.

- Chatterjee, A., Hu, X. y Lam, F. L. Y. (2018). Case Study 2: Development of Hydrothermally Stable Functional Materials for Sustainable Conversion of Biomassto Furan Compounds. En R. Luque y F. L. Y. Lam (Eds.), Sustainable Catalysis: Energy-Efficient Reaction and Applications (pp. 251272). Wiley-VCH Verlag GmbH \& Co Weinheim KGaA. doi: https://doi. org/10.1002/9783527693030.oth2.

- Coronado, M., Vega, S., Gutiérrez, R., Vázquez, M. y Radilla, C. (2015). Antioxidantes: perspectiva actual para la salud humana. Revista Chilena de Nutrición, 42(2), 206:212. doi: https://doi.org/10.4067/S0717-75182015000200014.

- Cruz, N., Bustos, C., Aguayo, M. G., Cloutier, A. y Castillo, R. (2018). Impact of the Chemical Composition of Pinus Radiata Wood on its Physical and Mechanical Properties Following Thermos-Hygromechanical Densification. BioResources, 13(2), 2268-2282. doi: https://doi.org/10.15376/biores.13.2.2268-2282.

- DANE. (2015). Encuesta Nacional Agropecuaria ena 2015. Bogotá D.C.: DANE.

- Del Val Gento, V. y Giraldo, G. A. (2016). Planta de cogeneración mediante gasificación de biomasa residual (Tesis de pregrado en Ingeniería Industrial). Escuela Técnica Superior de Ingenieros Industriales, Valladolid. Recuperado de http://www.eis.uva.es/energias-renovables/trabajos_07/CogeneracionBiomasa.pdf.

- Demirbas, A. (2010). Fuel from Biomass. En A. Demirbas (Ed.), Biorefineries for Biomass Upgrading Facilities (pp. 33-74). Londres: Springer.

- Do Nascimento, T., Damilano, D., Gomes, A., Bezerra, F. C., Rogrigues, R. F., Cordeiro, D. ... y Cezar, R. S. (2016). Potential for Biofuels from the Biomass of Prickly Pear Cladodes: Challenges for Bioethanol and Biogas Production in Dry Areas. Biomass and Bioenergy, 85, 215-222. doi: https://doi.org/10.1016/j. biombioe.2015.12.005.

- Galán, X. F. (2016). Potencial energético de la biomasa residual agrícola en Colombia (Tesis de especialización). Especialización en Gestión Ambiental, Fundación Universidad de América, Bogotá D.C.

- Gonzáles, K. D., Daza, D., Caballero, P. A. y Martínez, Ch. (2016). Evaluación de las propiedades físicas y químicas de residuos sólidos orgánicos a emplearse en la elaboración de papel. Luna Azul, 43, 499-517. doi: https://doi.org/ 10.17151/luaz.2016.43.21.

- González, R. (2002). Eugenol: propiedades farmacológicas y toxicológicas. Ventajas y desventajas de su uso. Revista Cubana de Estomatología, 39(2), 139-156. Recuperado de http://scielo.sld.cu/scielo.php?script=sci_ arttext\&pid=S0034-75072002000200005.

- Han, J. S. y Rowell, J. S. (1997). Chemical Composition of Fibers. En R. M. Rowell, R. A. Young y J. K. (Eds), Paper and Composites from Agro-Based Resourses (pp. 83-134). Boca Ratón: CRC press.

- Harshvardhan, K., Sura, M., Goswami, A. y Goswami, T. (2017). Biological Approach for the Production of Vanillin from Lignocellulosic Biomass (Bambusa tulda). Journal of Cleaner Production, 149, 485-490. doi: https://doi. org/10.1016/j.jclepro.2017.02.125.

- Hupa, M., Karlström, O. y Vainio, E. (2017). Biomass Combustion Technology Development-It is All About Chemical Details. Proceedings of the Combustion institute, 36(1), 113-134. doi: https://doi.org/10.1016/j.proci.2016.06.152.

- Jiménez-Mora, J. P. y Rodríguez-Romero, W. (2018). Actividad de enzimas antioxidants eritrocitarias en una muestra de adultos jóvenes y mayores del Valle Central, Costa Rica. Revista Biomédica, 29(1),13-24. Recuperado de http:// www.medigraphic.com/pdfs/revbio/bio-2018/bio181c.pdf.

- Kumar, A., Lee, Y., Kim, D., Rao, K. M., Kim, J., Park, S... y Han, S. S. (2017). Effect of Crossilinking Functionality of Microstructure, Mechanical Properties, and In Vitro Cytocompatibility of Cellulose Nanocrystals Reinforced Poly (Vinyl Alcohol)/Sodium Alginate Hybrid Scaffolds. International Journal of Biological Macromolecules, 95, 962-973. doi: https://doi.org/10.1016/j.jibiomac.2016.10.085.
- Londoño-Londoño, J., Sierra, J., Álvarez, R., Restrepo, A. M. y Pássaro C. P. (2012). Aprovechamiento de los subproductos citrícolas. L. F. Garcés (Ed.), Cítricos: cultivo, porcosecha e industrialización (pp. 343-367). Caldas: Corporación Universitaria Lasallista.

- Maeda, N., Katakura, T., Fukasawa, T., Huang, A. N., Kawano, T. y Fukui, K. (2017). Morphology of Woody Biomass Combustion Ash and Enrichment of Potassium Components by Particle Size Classification. Fuel Processing Technology, 156, 1-8. doi: https://doi.org/10.1016/j.fuproc.2016.09.026.

- Maneerun, T., Kawi, S. y Wang, ChH. (2015). Biomass Gasification Bottom Ash as A Source of Cao Catalyst for Biodiesel Production Via Transesterification of Palm Oil. Energy Conversion and Management, 92, 234-243. doi: https://doi. org/10.1016/j.enconman.2014.12.057.

- Martínez-Valverde, I., Periago, M. J. y Ros, G. (2000). Significado nutricional de los compuestos fenólicos de la dieta. Archivos Latinoamericanos de Nutrición, 50(1), 5-18.

- Mejía, L. F., Martínez, H. A., Betancourt, J. E. y Castrillón, C. E. (2007). Aprovechamiento del residuo agroindustrial del mango común (Mangifera indica L.) en la obtención de azúcares fermentables. Ingeniería y Ciencia, 3(6), 41-62. Recuperado de http://publicaciones.eafit.edu.co/index.php/ingciencia/article/view/440.

- Melissari, B. (2012). Comportamiento de cenizas y su impacto en sistemas de combustión de biomasa. Memoria de Trabajos de Difusión Científica y Técnica, 10, 69-82. Recuperado de http://www.um.edu.uy/docs/6 comportamiento de_cenizas_y_suimpacto_en_sistemas_de_\%20combustion_de_biomasa.pdf.

- Ministerio de Agricultura y Desarrollo Rural. (2006). Plan Frutícola NacionalDesarrollo de la fruticultura en Caldas. Gobernación de Caldas, Manizales.

- Muñoz-Muñoz, D., Pantoja-Matta, A. J. y Cuatín-Guarín, M. F. (2014). Aprovechamiento de residuos agroindustriales como biocombustible y biorefinería. Biotecnología en el Sector Agropecuario y Agroindustrial, 12(2), 10-19. http:// www.scielo.org.co/pdf/bsaa/v12n2/v12n2a02.pdf.

- Panesso, E. A., Calle, M. C. y Meneses, E. J. (2012). Salud bucal y xilitol: usos y posibilidades en caries y enfermedad periodontal en poblaciones "pepe". Revista Universidad y Salud, 14(2), 205-215. Recuperado de http://www.scielo.org.co/ scielo.php?script=sci_arttext\&pid=S0124-71072012000200010\&lng=en\&nrm=iso.

- Pedraja, A. (2016). Combustión, gasificación y pirólisis de lignosulfonato de calcio mediante tg-dsc-ms (Tesis de pregrado en Técnico Industrial). Universidad de Cantabria, Cantabria. Recuperado de http://hdl.handle.net/10902/8166.

- Peñaranda, L. V., Montenegro, S. P. y Giraldo, P. A. (2017). Aprovechamiento de residuos agroindustriales en Colombia. Revista de Investigación Agrariay Ambiental, 8(2), 141-150. doi: https://doi.org/http://dx.doi.org/10.22490/21456453.2040.

- Pérez, Á. T., Batista, M. A., Velásquez, J. C. y García, J. M. (2013). Acetato de celulosa del bagazo de la caña de azúcar: plastificación y evaluación de propiedades. Ciencias Holguín, 19(4), 1-9. Recuperado de http://www.ciencias. holguin.cu/index.php/cienciasholguin/article/view/818/846.

- Pradyawong, S., Qi, Q., Li, N., Sun, X. S. y Wang, D. (2017). Adhesion Properties of Soy Protein Adhesives Enhanced by Biomass Lignin. International Journal of Adhesion and Adhesives, 75, 66-73. doi: https://doi.org/10.1016/j. ijadhadh.2017.02.017.

- Prinsen, P., (2010). Composición química de diversos materiales lignocelulósicos de interés industrial y análisis estructural de sus ligninas (Tesis de maestría). Maestría en Estudios Avanzados en Química, Universidad de Sevilla, Sevilla. doi: https://doi.org/10.7868/S0555109913030112.

- Ramírez, K., Rojas, Ó., Alvarado, P. y Vega-Baudrit, J. (2012). Obtención de xilosa a partir de desechos lignocelulósicos de la producción y proceso industrial de la piña (Ananas comusus). Uniciencia, 26, 75-89. Recuperado de https://biblat.unam.mx/es/revista/uniciencia/articulo/obtencion-de-xilosa-apartir-de-desechos-lignocelulosicos-de-la-produccion-y-proceso-industrialde-la-pina-ananas-comosus.

- Rentería, J. L. (2014). Procesamiento de frutas de maracuyá (Passiflora edulis) para obtención de pectina, en Machala, 2013 (Tesis de pregrado). Programa de Ingeniería Agronómica, Universidad Técnica De Machala, Machala. Recuperado de http://repositorio.utmachala.edu.ec/handle/48000/1045.

- Ruales, Á. V. (2015). Evaluación del potencial energético y bioactivo de los residuos generados por la producción y transformación de la uva (Tesis 
de maestría). Maestría en Ingeniería Química, Universidad Nacional de Colombia, Manizales.

- Sánchez A. (2016). Modelado del proceso de extracción de ácido acético con recuperación del disolvente orgánico (Tesis de pregrado). Maestría en Ingeniería Química, Universidad Politécnica de Madrid, Madrid. Recuperado de http://oa.upm.es/42845/1/TFG_ANA_SANCHEZ_LEVOSO.pdf.

- Sánchez-Mendoza, N. A, Cruz-Castellanos, M., Dávila-Ortiz, G. y JiménezMartínez, C. (2016). Péptidos con actividad antioxidante provenientes de fuentes animales y vegetales. M. E. Ramírez (Ed.), Propiedades funcionales de hoy (pp. 117-14). Barcelona: OmniaScience. Recuperado de http:// www.omniascience.com/monographs/index.php/monograficos/article/ view/352/254.

- Sandak, A., Sandak, J., Waliszewska, B., Zborowska, M. y Mleczec, M. (2017). Selection of Optimal Conversion Path for Willow Biomass Assisted by Infrared Spectroscopy. iForest Biogeosciences and Forestry, 10, 506-514. doi: http:// doi/10.3832/ifor1987-010.

- Sanz, A. (2016). Tecnología de la celulosa. La industria papelera. Escuela de Ingenierías Industriales-Uva (España). Recuperado de https://www.eii.uva.es/ organica/qoi/tema-03.php, consulta octubre de 2017.

- Saval, S. (2012). Aprovechamiento de residuos agroindustriales: pasado, presente y futuro. BioTecnología, 16(2), 14-16. Recuperado de https://smbb. $\mathrm{mx} / \mathrm{wp}$-content/uploads/2017/10/Revista_2012_V16_n2.pdf\#page=14.

- Shokri, J. y Adibkia, K. (2013). Application of Cellulose and Cellulose Derivatives in Pharmaceutical Industries. En T. Van De Ven y L. Godbout (Eds.), Cellulose-Medical, Pharmaceutical and Electronic Applications (pp. 47-66). Irán: InTech. doi: https://doi/10.5772/55178.

- Suárez, N. (2010). Estudio de la sustitución parcial del fenol por lignina en la obtención de las resinas fenol-formaldehído utilizadas para la fabricación de tableros aglomerados osb a partir de especies forestales colombianas (Tesis de pregrado). Programa de Química Industrial, Universidad Tecnológica de Pereira, Pereira. Recuperado de http://hdl.handle.net/11059/2051.

- V. K., Gupt, Carrot, P. J. M., Singh, R., Chaudhary, M. y Kuchwaha, S. (2016). Cellulose: A Review as Natural, Modified and Activated Carbon Adsorbent, Suhas. Bioresource Technology, 216, 1066-1076. doi: https://doi.org/10.1016/j. biortech.2016.05.106.

- Trapero, Y. M. (2013). Farmacocinética y toxicología del 4-hidroxi-3-metoxibenzaldehído (vainillina) en modelos in vivo (Tesis de doctorado en Ciencias de la Salud). Universidad de Oriente, La Habana. Recuperado de http://tesis.sld. cu/index.php?P=FullRecord\&ID=254.

- Vargas, Y. A.y Pérez, L. I. (2018). Aprovechamiento de residuos agroindustriales en el mejoramiento de la calidad del ambiente. Revista Facultad de Ciencias Básicas, 14(1), 1-14. Recuperado de https://revistas.unimilitar.edu.co/index. $\mathrm{php} / \mathrm{rfcb} /$ article/view/3108/2874.

- Yepes, S. M., Montoya, L. J. y Orozco, F. (2008). Valorización de residuos agroindustriales -frutas - en Medellín y el sur del Valle del Aburrá, Colombia. Revista de la Facultad Nacional de Agronomía Medellín, 61(1), 44224431. Recuperado de http://www.redalyc.org/pdf/1799/179914077018.pdf. 